\title{
Ruptura do Septo Interventricular após Trauma Torácico Fechado
}

\author{
Ventricular Septal Rupture Following Blunt Chest Trauma \\ Ronaldo Peixoto de Mello, Maria Virginia Santana, Maria Aparecida de Paula Silva, César Esteves, Carlos Pedra, \\ Anneliese Thom, Paulo Chaccur, Marly Akiko Miaira, Pedro Beraldo Andrade, Alejandra Borges Rodrigues \\ Instituto Dante Pazzanese de Cardiologia - São Paulo, SP
}

Relatamos a seguir um caso de ruptura do septo interventricular após trauma torácico automobilístico com evolução relativamente benigna a despeito da extensão da lesão anatômica.
We report a case of ventricular septal rupture following chest trauma resulting from a car accident. The outcome was relatively favorable despite the extension of the anatomic lesion.
A contusão cardíaca representa a forma mais comum de trauma cardíaco não penetrante, sendo responsável pela gênese da ruptura de septo interventricular (RSIV) adquirido nessa condição. Esta foi primeiramente descrita por Hewett em $1847^{1}$. Entretanto, o trauma torácico não penetrante é uma causa rara de RSIV, habitualmente de evolução não fatal, sendo associada a impactos de alta energia, e com freqüência não diagnosticada precocemente porque negligenciada ${ }^{2}$.

\section{Relato do Caso}

\section{Ananmese}

Adolescente de 18 anos sexo masculino, $75 \mathrm{~kg}$, sem antecedentes prévios, relatando acidente automobilístico recente (40 dias antes da internação), seguido de traumatismo crânio-encefálico e trauma torácico contuso. Atendido em serviço de emergência, evoluiu na ocasião com perda prolongada da consciência e contusões pulmonares múltiplas que não necessitaram de intervenção, recebendo alta hospitalar após observação mínima. Nessa ocasião, referia estar assintomático do ponto de vista cardiovascular. No período de convalescença, após 10 dias do evento, relatou o aparecimento rápido e progressivo de dispnéia aos médios esforços associados a vibração torácica intensa que acompanhava o ritmo cardíaco. Procurou atendimento médico, tendo sido diagnosticado com sopro cardíaco e logo encaminhado a este serviço. Na consulta inicial apresentava dispnéia para caminhar 100 metros e grande dificuldade para transpor terrenos inclinados. Isso o obrigava a inúmeras interrupções no trajeto, associado a incapacidade marcante para o desempenho funcional, porém, mantinha-se sem prejuízo na tolerância ao decúbito. Negava na ocasião palpitação, dor torácica, tontura ou dispnéia aos pequenos esforços. Informava uso regular de maconha e bebidas alcoólicas; negava uso de drogas injetáveis ou cocaína.

\section{Exame físico}

Pressão arterial 120/70 mmHg em membros superiores, pulso 80 bpm, em bom estado geral, corado, hidratado, acianótico, sem baqueteamentos. Exame pulmonar sem ruídos adventícios com murmúrio vesicular universalmente audível. Aparelho cardiovascular evidenciou ictus cordis hipercinético, frêmito palpável $++/ 4$ na borda esternal esquerda baixa, sopro cardíaco $+++/ 4$, holossistólico, regurgitativo, na borda esternal esquerda com irradiação para a direita. Segunda bulha com desdobramento fisiológico e normofonética. Abdome sem hepatomegalias, ruídos hidroaéreos preservados, indolor e sem defesas. Pulsos palpáveis, simétricos e normais.

\section{Exames complementares}

Eletrocardiograma, radiografia torácica, ecocardiograma e desenho esquemático podem ser avaliados nas figuras 01 a 04. Na ocasião do diagnóstico, o ecocardiograma bidimensional evidenciou ampla RSIV muscular trabecular, com extensão para região infundibular, $\mathrm{FE}=0,74$, diâmetro sistólico do ventrículo esquerdo (DSVE) de $39 \mathrm{~mm}$ e diâmetro diastólico do ventrículo esquerdo (DDVE) $70 \mathrm{~mm}$. Cateterismo cardíaco esquerdo e direito confirmou a suspeita diagnóstica de RSIV, evidenciando amplo shunt esquerda-direita. As medidas de pressão (tab. 01) evidenciaram hiperfluxo pulmonar importante sem hipertensão pulmonar associada (relação $\mathrm{Rp} / \mathrm{Rs}=0,05$ e Qp/Qs= 4:1).

Corrigida a ruptura do septo interventricular, através de esternotomia mediana, com perfusão e proteção miocárdica por cardioplegia cristalóide. Identificada cardiomegalia à custa de átrio direito, ventrículo direito e tronco pulmonar, e RSIV de aproximadamente $3 \mathrm{~cm}$ localizado abaixo da banda moderadora na porção mediana do septo. Realizado ventriculosseptoplastia com patch de pericárdio bovino utilizando 15 fios de ethibond 2.0 (pontos em U) ancorados com teflon. No pós-operatório imediato evoluiu com sangramento cirúrgico necessitando de revisão de

\section{Palavras-chave}

Ruptura do septo interventricular, traumatismos cardíacos, comunicação interventricular. 


\section{Relato de Caso}

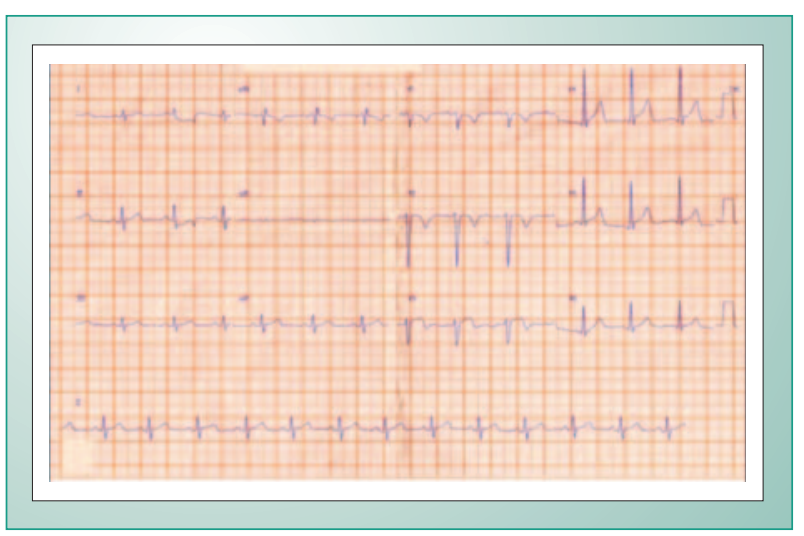

Fig. 1 - Eletrocardiograma de repouso evidencia ritmo sinusal, eixo 60 graus, sem sobrecargas, progressão lenta de onda R e inversão permanente de onda T de V1 a V3.

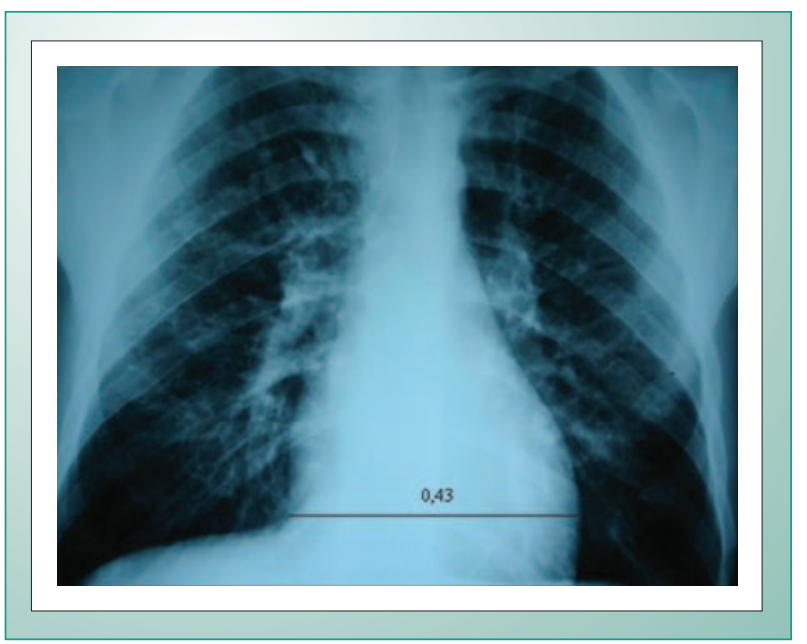

Fig. 2 - Radiografia torácica: observa-se situs solitus, área cardíaca com diâmetros preservados (índice Danzer de 0,43), fluxo pulmonar aumentado $++++/ 4$ e tronco da pulmonar retificado.

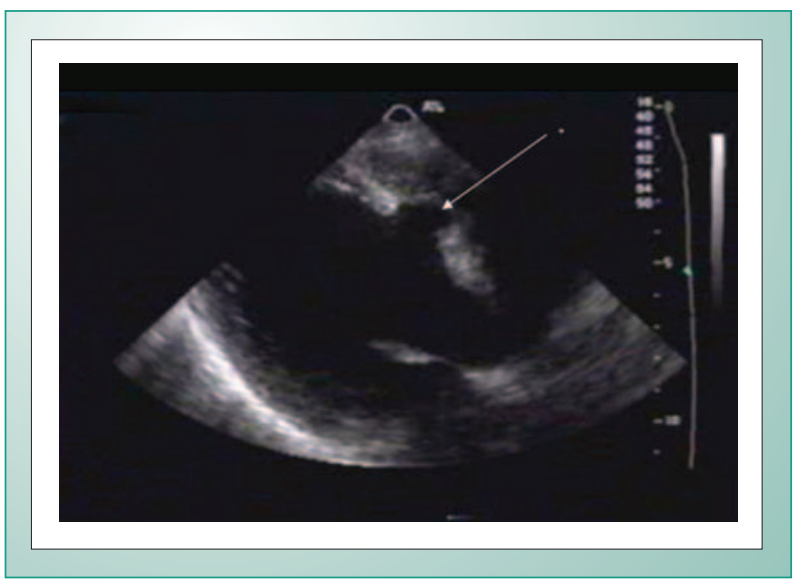

Fig. 3 - Ecodopplercardiograma mostrou situs solitus vícero-atrial com levocardia e levoposição do ápex, presença de forame oval patente com shunt esquerda-direita e ampla comunicação interventricular muscular trabecular (*) com extensão para região infundibular medindo $30 \mathrm{~mm}$, com shunt esquerda-direita. Aumento moderado do ventrículo esquerdo com função sistólica preservada.

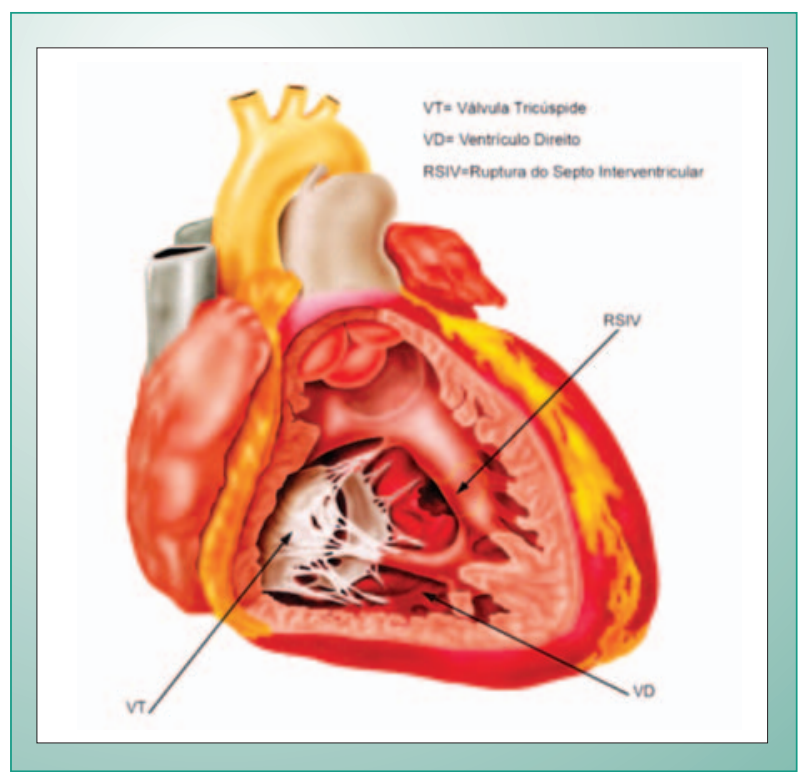

Fig. 4 - RSIV de aproximadamente $3 \mathrm{~cm}$ localizado abaixo da banda moderadora na porção mediana do septo.

\begin{tabular}{|lcc|}
\hline & $\begin{array}{c}\text { Pressões } \\
\text { (mmHg) }\end{array}$ & $\begin{array}{c}\text { Saturação de } \\
\text { O2 (\%) }\end{array}$ \\
\hline Veia cava superior & 8 & 69 \\
\hline Átrio direito (média) & 75 \\
\hline Ventrículo direito & $60 / 8(25)$ & 90 \\
\hline Artéria pulmonar & $45 / 13(26)$ & 92 \\
\hline Ventrículo esquerdo & $110 / 15(46)$ & 99 \\
\hline Aorta & $90 / 50(63)$ & 97 \\
\hline \multicolumn{2}{|c}{ Tabela 1 - Dados do cateterismo cardíaco } \\
\hline
\end{tabular}

hemostasia. Atualmente, encontra-se em acompanhamento ambulatorial regular, assintomático, em classe funcional I. Ecocardiograma bidimensional de controle no terceiro mês de pós-operatório evidenciou FE de 0,72 e regressão importante da cavidade ventricular esquerda para DDVE de $55 \mathrm{~mm}$ além da presença de RSIV residual na porção médio-muscular septal medindo $3 \mathrm{~mm}$. Radiografia torácica de controle sem hiperfluxo.

\section{Discussão}

A contusão cardíaca que culmina com a evolução para RSIV pode ser conseqüência de vários fatores que resultam da interação entre as forças que agem sobre o tecido cardíaco no momento do impacto. Parmley classificou as causas de injúria cardíaca em 7 categorias: (a) forças de ação direta, (b) forças indiretas, (c) forças bidirecionais ou compressivas, (d) desaceleração, (e) forças explosivas, (f) concussão e (g) a combinação destas, seja agindo diretamente sobre o tórax ou dorso, como conseqüência da alteração brusca do movimento linear pela desaceleração ou mudança abrupta deste ${ }^{2,3}$.

A compressão empregada pela caixa torácica sobre o coração, mais especificamente entre o esterno e a coluna torácica com resultante aumento abrupto da pressão 
intracardíaca durante a diástole tardia ou sístole isovolumétrica, quando as câmaras cardíacas estão cheias e as valvas estão fechadas, impossibilita o alívio imediato da pressão interna, constituindo o principal evento na gênese da contusão tecidual e da ruptura. A lesão coronariana secundária à contusão, entretanto, constitui outro mecanismo possível e poderia ser responsabilizada pelos casos de apresentação clínica tardia, como conseqüência de infarto, necrose local e posterior ruptura muscular ${ }^{3-5}$.

A verdadeira incidência do RSIV após trauma torácico, assim como a participação dos agentes etiológicos em particular, permanece desconhecida na literatura. Entretanto, além do trauma contuso fechado, lesões penetrantes, lesões coronarianas agudas, endocardite bacteriana e as iatrogênicas decorrentes de procedimentos diagnósticos ou terapêuticos têm sido descritas como formas adquiridas de RSIV. Publicações prévias avaliando autópsia de 207.548 casos de óbito secundário a acidente automobilístico identificaram RSIV em 30 casos $(0,01 \%)$, sendo que em apenas 0,002\% os defeitos se apresentaram de forma isolada. Nesta série, a ruptura da parede livre ventricular foi a forma mais comum de apresentação ${ }^{1,6}$. Por outro lado, séries clínicas têm variado a incidência entre $10 \%$ a $75 \%$ na presença de trauma torácico intenso ${ }^{2}$. A presença de contusão miocárdica tem sido estimada em $15 \%$ das vítimas de trauma torácico fatal avaliados em séries de autopsia ${ }^{7}$. No Brasil, por exemplo, em 2002, ocorreram 33.288 mortes por acidente automobilístico de acordo com dados do Datasus ${ }^{8}$; destas, 22.928 mortes ocorreram em indivíduos entre 15 e 49 anos de idade. Somente no estado de São Paulo foram 1.046 mortes, em 2000, entre indivíduos na faixa etária de 15 a 24 anos. Muitos destes indivíduos faleceram secundariamente a traumas múltiplos, entre eles traumatismo craniano e/ou torácico. Apesar da ausência de dados confiáveis quanto à incidência das complicações cardíacas relacionadas a trauma torácico fechado, tais números podem nos oferecer condições de avaliar a dimensão do problema, especialmente se levarmos em consideração que, na revisão feita por Parmley, a ruptura cardíaca foi a forma mais comum de injúria, ocorrendo em $64 \%$ dos $\operatorname{casos}^{2,6}$.

No presente caso, inúmeros elementos corroboram o diagnóstico de origem traumática do defeito septal: (a) história clínica prévia negativa para sintomas cardíacos, lembrando a natureza da atividade profissional do paciente, (b) eletrocardiograma sem sinais de sobrecarga ventricular e (c) radiografia de tórax evidenciando área cardíaca sem prejuízo, incompatíveis com a extensão do RSIV avaliada pelo ecocardiograma. Campbell, ao estudar a história natural do defeito congênito, demonstrou que $27 \%$ dos indivíduos portadores de grandes defeitos morrem por disfunção ventricular e hipertensão pulmonar secundária antes dos 20 anos de idade e 53\% antes dos 40 anos, se não forem tratados adequadamente ${ }^{9}$. Tais dados sugerem ser remota a possibilidade de este paciente ter evoluído até a adolescência sem diagnóstico prévio ou evolução fatal para insuficiência cardíaca.

Para favorecer o diagnóstico é necessário um alto índice de suspeita clínica, visando prevenir primariamente as potenciais conseqüências letais da ruptura ventricular. Inúmeros métodos estão disponíveis na atualidade, tais como ECG, marcadores enzimáticos, cintilografia de perfusão miocárdica e ecocardiograma (convencional ou transesofágico).

A forma de apresentação clínica pode ser aguda, subaguda ou tardia, na dependência da magnitude da injúria e conseqüente necrose local $^{2}$. Na revisão feita por Rotman e cols. ${ }^{3}$, o sopro cardíaco esteve inicialmente presente em poucos casos, sendo que na maioria tornou-se aparente entre 4 e 12 dias, enquanto que o surgimento de mais de um ponto de ruptura foi apresentação incomum. Os sintomas têm um amplo espectro clínico. Podem variar desde casos assintomáticos, dor torácica atípica, dor anginosa, dispnéia ou palpitação, até quadros mais graves de insuficiência cardíaca progressiva, choque cardiogênico e morte. Chama a atenção a apresentação clínica rapidamente progressiva para dispnéia aos moderados esforços (CF II) no presente caso, sem evolução para dispnéia de decúbito ou insuficiência cardíaca franca a despeito do grande defeito septal. Rotman e cols. ${ }^{3}$, ao analisarem esta questão, advertem que o espectro sintomático pode não estar direcionado necessariamente ao aparelho cardiovascular, podendo ser dominado pelo comprometimento de outros órgãos atingidos com conseqüente surgimento dos sintomas cardiovasculares após o retorno às atividades normais no período de convalescença. Nesse caso, ao revisar 38 casos de RSIV traumática, encontrou 08 indivíduos assintomáticos e 24 indivíduos com sintomas compatíveis com insuficiência cardíaca, dispnéia ou palpitações, sendo que a dor torácica anginosa, a insuficiência cardíaca rapidamente progressiva, o choque cardiogênico e morte foram apresentações menos comuns.

Os sinais detectados ao exame clínico são semelhantes aos do defeito congênito. A radiografia torácica evidencia habitualmente cardiomegalia, hiperfluxo pulmonar e/ou congestão pulmonar proporcionais ao grau do defeito ${ }^{3}$.

As alterações eletrocardiográficas são inespecíficas para contusão e incluem anormalidades do segmento ST e da onda T. Arritmias ventriculares e atriais podem estar presentes, sendo raro o relato de bloqueio atrioventricular ${ }^{3}$. Apesar de útil na avaliação inicial, tem baixa sensibilidade e especificidade, mascarados especialmente por alterações inespecíficas e/ou prévias. Em uma série com 50 pacientes, a dosagem sérica seriada de CPK/CKMB esteve alterada em 20\% dos pacientes estudados, detectando apenas 50\% dos casos de injúria cardíaca posteriormente diagnosticada por ecocardiograma transtorácico. Nessa série, a relação CPK/CKMB obteve sensibilidade e especificidade de $10 \%$ e $77,5 \%$, respectivamente ${ }^{10}$.

O ecocardiograma transtorácico é um método útil, podendo identificar déficit da movimentação segmentar, dilatação de câmaras, além de avaliar a extensão da lesão e espessura miocárdica. É capaz de oferecer informações importantes quanto à função ventricular direita e esquerda e excluir potenciais complicações como hematomas, tamponamentos, entre outras. Porém, por influências diversas, dificuldades técnicas podem estar presentes em cerca de $20 \%$ dos casos $^{3}$, além de possuir valor limitado na avaliação da motilidade ventricular direita, bem como no estudo do trato de saída deste. Weiss e cols. ${ }^{10}$, estudando 22 pacientes com diagnóstico pelo ecocardiograma transesofágico de contusão miocárdica, identificou que apenas $27 \%$ dos pacientes tinham ECG altamente sugestivo, e advoga o uso rotineiro deste método para todos os pacientes com suspeita clínica. Em seu estudo, a detecção de sinais compatíveis com contusão miocárdica esteve associada a significativa mortalidade. O ecocardiograma transesofágico tem a vantagem de ser uma técnica minimamente invasiva, avalia de forma localizada anormalidades da motilidade e funções ventriculares tanto esquerda quanto direita ${ }^{10}$. A decisão terapêutica quanto ao tratamento cirúrgico segue os mesmos parâmetros utilizados para o defeito congênito. 


\section{Relato de Caso}

\section{Referências}

1. Rutherford EJ, White KS, Maxwell JG, Clancy TV. Immediate isolated interventricular septal defect from nonpenetrating thoracic trauma. Am Surg. 1993; 59: 353-4.

2. Stahl RD, Liu JC, Walsh JF. Blunt cardiac trauma: atrioventricular valve disruption and ventricular septal defect. Ann Thorac Surg. 1997; 64:14668.

3. Rotman M, Peter RH, Sealy WC, Morris JJ Jr. Traumatic ventricular septal defect secondary to nonpenetrating chest trauma. Am J Med. 1970; 48: 127-31.

4. Aris A, Delgado LJ, Montiel J, Subirana MT. Multiple intracardiac lesions after blunt chest trauma. Ann Thorac Surg. 2000; 70: 1692-4.

5. Stajer D, Kariz S. Ventricular septal rupture following blunt chest trauma after a long delay: a case report. Int J Cardiol. 1994; 47: 187-8.
6. Parmley LF, Manion WC, Mattingly TW. Nonpenetrating traumatic injury of the heart. Circulation. 1958; 18: 371-96.

7. Ministério da Saúde [homepage na Internet]. Secretaria Executiva. Datasus [citado 2005 maio 6]. Disponível em: http://www.datasus.gov.br.

8. Campbell M. Natural history of ventricular septal defect. Br Heart J. 1971; 33: 246-57.

9. Lidenbaum GA, Carroll SF, Block EF, Kapusnick RA. Value of creatine phosphokinase isoenzyme determinations in the diagnosis of myocardial contusion. Ann Emerg Med. 1988; 17: 885-9.

10. Weiss RL, Brier JA, O'Connor W, Ross S, Brathwaite CM. The usefulness of transesophageal echocardiography in diagnosing cardiac contusions. Chest. 1996; 109: 73-7. 